WellBeing International

WBI Studies Repository

$12-2011$

\title{
Lessons from Chimpanzee-based Research on Human Disease: The Implications of Genetic Differences
}

Jarrod Bailey

New England Anti-Vivisection Society

Follow this and additional works at: https://www.wellbeingintlstudiesrepository.org/acwp_lab

Part of the Animal Experimentation and Research Commons, Animal Studies Commons, and the Other Medical Sciences Commons

\section{Recommended Citation}

Bailey, J. (2011). Lessons from chimpanzee-based research on human disease: the implications of genetic differences. Alternatives to Laboratory Animals-ATLA, 39(6), 527.

This material is brought to you for free and open access by WellBeing International. It has been accepted for inclusion by an authorized administrator of the WBI Studies Repository. For more information, please contact wbisr-info@wellbeingintl.org.

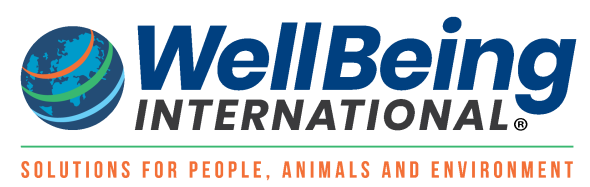




\title{
Lessons from Chimpanzee-based Research on Human Disease: The Implications of Genetic Differences
}

\author{
Jarrod Bailey \\ New England Anti-Vivisection Society (NEAVS), Boston, USA and British Union for the Abolition of \\ Vivisection (BUAV), London, UK
}

\begin{abstract}
Summary - Assertions that the use of chimpanzees to investigate human diseases is valid scientifically are frequently based on a reported 98-99\% genetic similarity between the species. Critical analyses of the relevance of chimpanzee studies to human biology, however, indicate that this genetic similarity does not result in sufficient physiological similarity for the chimpanzee to constitute a good model for research, and furthermore, that chimpanzee data do not translate well to progress in clinical practice for humans. Leading examples include the minimal citations of chimpanzee research that is relevant to human medicine, the highly different pathology of HIV/AIDS and hepatitis C virus infection in the two species, the lack of correlation in the efficacy of vaccines and treatments between chimpanzees and humans, and the fact that chimpanzees are not useful for research on human cancer. The major molecular differences underlying these inter-species phenotypic disparities have been revealed by comparative genomics and molecular biology - there are key differences in all aspects of gene expression and protein function, from chromosome and chromatin structure to post-translational modification. The collective effects of these differences are striking, extensive and widespread, and they show that the superficial similarity between human and chimpanzee genetic sequences is of little consequence for biomedical research. The extrapolation of biomedical data from the chimpanzee to the human is therefore highly unreliable, and the use of the chimpanzee must be considered of little value, particularly given the breadth and potential of alternative methods of enquiry that are currently available to science.
\end{abstract}

Key words: animal model, chimpanzee, genetics, Pan troglodytes.

Address for correspondence: Jarrod Bailey, NEAVS, 333 Washington Street, Suite 850 Boston, MA 02108-5100, USA; BUAV, 16a Crane Grove, London N7 8NN, UK. E-mail: jarrod.bailey@mac.com

\section{Introduction}

The validity of using chimpanzees (Pan troglodytes) in research to investigate the diseases that affect humans is frequently based on the reported genetic similarity of the two species. It is often noted that chimpanzees are $98-99 \%$ genetically identical to humans - a figure that was initially derived 40 years ago $(1,2)$ and was confirmed more recently by using superior technologies (3). Advocates of experimentation with chimpanzees claim it is axiomatic that this genetic similarity leads to equivalent biological and physiological similarity, and that because chimpanzees are the closest relatives of humans, they must constitute a good - indeed the best - animal species to use for the study of human disease (4). Thus, the use of chimpanzees in biomedical research is justified by its proponents, alongside claims of the crucial role played by such research in fighting many grave human diseases.

However, this justification has become increasingly controversial. Given the cognitive and emotional capacities of chimpanzees, and the harmful consequences of laboratory life and invasive research for the animals involved $(5,6)$, profound ethical considerations have always been the foundation for strong opposition to chimpanzee research $(7,8)$. In addition, in recent years a burgeoning scientific case has been made against the use of chimpanzees in research, which seriously questions the usefulness and human relevance of chimpanzee data (9-13; and see http://www. releasechimps.org/pdfs/chimp-efficacy-paper-main. pdf and http://www.releasechimps.org/pdfs/ChimpCA-suppl.pdf). Furthermore, it is apparent that the claimed 98-99\% genetic similarity between humans and chimpanzees is superficial and deceptive. The overall similarity, when all relevant factors are taken into account, is likely to be nearer to $95 \%(14,15)$, or even approaching $93 \%(16,17)$. As elaborated in this paper, important differences at all levels of gene expression and protein function, from chromosome structure to post-translational modification, combine to generate significant and widespread biological and physiological differences.

In recent years, such concerns have led to legislation that bans, or at least very severely restricts, chimpanzee experimentation in the relatively few 
countries where it was ever practised (see http:// www.releasechimps.org/mission/end-chimpanzeeresearch/country-bans/). The exception is the USA, where approximately 1,000 chimpanzees remain in laboratories (see http://www.releasechimps.org/ mission/end-chimpanzee-research/), many of them the last survivors of an intensive breeding programme for HIV/AIDS research that began in 1986 (18). The ethical and scientific concerns that have been raised have been reflected in substantial public opposition to chimpanzee experimentation (see http://www.releasechimps.org/mission/endchimpanzee-research/public-opinion/), which has translated into some degree of legal protection: the passage of the Chimpanzee Health, Improvement, Maintenance and Protection (CHIMP) Act in 2000 $(19,20)$, and the more-recent legislative efforts in both the US House of Representatives and the US Senate in the form of the Great Ape Protection Act (GAPA) in 2009 (21, 22), which was reintroduced in 2011 as the Great Ape Protection and Cost Savings Act (GAPCSA; H.R.1513/S.810; 23, 24). This act would end invasive experimentation on captive chimpanzees in the USA, bringing the country into line with the rest of the world, and would facilitate the retirement of all federally owned chimpanzees into permanent sanctuaries.

The aim of the present review is to aid the debate and discussion surrounding the use of chimpanzees in US laboratories, by examining the 'genetic similarity' argument in the light of current knowledge regarding the comparative molecular biology of humans and chimpanzees and their inter-species differences. Salient information from recent years, along with a consideration of the performance and human relevance of the chimpanzee model, is synthesised, in order to illuminate and critically evaluate this argument.

\section{Methods}

Papers describing important genetic differences between humans and chimpanzees, which have significant or potentially significant functional biological consequences, were located via the GoPubMed database (25). Other salient reports were located in two exceptional reviews of human/chimpanzee comparative genomics that were published in 2001 (26) and 2006 (27). A total of 178 papers, dating from the past decade (2001-2010), were examined to form the basis of a review, the aim of which is to illustrate, with a sound and comprehensive basis, that there are crucial inter-species differences with extensive and far-reaching effects. These differences permeate all aspects of gene expression and protein function, from chromosome and chromatin structure all the way through to post-translational modification.

\section{Results}

\section{Cytogenetic differences: Fusions, inversions and translocations}

There are numerous well-known structural differences between the human and chimpanzee genomes. For example, human chromosome 2 results from a fusion of ancestral chromosomes corresponding to chimpanzee chromosomes 12 and 13 (28). Compared to chimpanzees, human chromosomes 1 and 18, as well as others, contain large inverted stretches, and many human chromosomes contain large translocated or rearranged segments (26). These rearrangements exert a significant influence on the expression of the affected genes via the 'position effect', which results from an alteration in the location of a gene. The relocation of a gene can influence its expression via changes in the proximity and/or nature of cis-acting promoters and enhancers. Furthermore, the local structural environment of chromatin can alter the accessibility of transcriptional proteins, and can exert gene-silencing effects via the influence of nearby heterochromatic DNA (29). One study 'conservatively estimated' that $18 \%$ of genes numbering some 3,200 - have genomic neighbourhoods that differ between humans and chimpanzees, and may therefore be subject to gene expression changes due to position effects (30).

Another major difference is found between the $\mathrm{Y}$ chromosomes of humans and chimpanzees. The male-specific region (MSY) of each of these chromosomes has evolved rapidly over the past six million years, and as a result, the chromosomes now 'differ radically in sequence, structure and gene content' (31). The chimpanzee MSY contains twice as many massive palindromes as its human counterpart and has lost genes, while the human MSY has gained genes. X-degenerate regions of the chimpanzee Y chromosome have lost four out of 16 genes, due to inactivating mutations. In total, the chimpanzee MSY contains only two-thirds of the number of genes/gene families in the human MSY, and just $50 \%$ of the protein-coding transcription units (31).

\section{Mobile DNA elements and copy number variation}

The position effect can also be invoked by the action of 'mobile elements' or 'transposable elements' in the genome - sections of DNA that can move or 'transpose' themselves to other genomic locations. One subclass of these elements can be classified as long interspersed nuclear elements (LINEs) and short interspersed nuclear elements (SINEs; 26); these vary between humans and 
chimpanzees and cause variations in gene expression between them. Mobile elements comprise almost half of the entire human genome $(26,32)$, and significantly affect gene function by physically disrupting genes, by generating alternative sites for the splicing of RNA molecules during gene transcription, or by interfering with gene promoter and enhancer regions (33). Among other things, they are responsible for an as yet undefined proportion of large duplicated regions of DNA called segmental duplications, which in total comprise a significant $5 \%$ of the human genome. While approximately two-thirds of these duplications are common to humans and chimpanzees, one-third are species-specific and have contributed greatly to the biological differences between humans and chimpanzees (34).

These duplications, in a phenomenon known as copy number variation, are responsible for significant disparities in gene expression and subsequent biological consequences, such as susceptibility to disease (35). They are known to be one of the most significant causes of genetic variation among primates (36), and are at the root of many aspects of intra-species and inter-species diversity, differentially affecting many human and chimpanzee genes involved in critical processes, such as immune and inflammatory responses, cell proliferation and tumour formation (37). Clearly, copy number variation greatly affects the appropriateness of chimpanzee use in the study of human diseases.

In 2006, Bailey and Eichler reported that over 76 million base pairs of DNA are differentially duplicated between chimpanzees and humans (35). More recently, Armengol and co-workers examined over 16,000 genes and identified 23 human-specific copy number differences (CNDs) versus chimpanzees, and 26 chimpanzee-specific CNDs versus humans, with some of the affected genes having neuronal functions (36). An earlier analysis revealed 177 complete and partial gene duplications in humans, but not in chimpanzees; the opposite was true for another 94 genes (34). Half of the 271 genes showed differences in expression - in fact, genes in duplicated regions are often up to 10 times more likely to show differences in expression levels than those in other regions (35). It is axiomatic that many genes with important functions would be affected: the multi-organ cancer susceptibility gene $C H E K 2$, for example, is present in nine copies in chimpanzees, but there are 13-16 copies in a typical human genome (38).

Inversions of genetic material are also highly prevalent, and are different, in the human and chimpanzee genomes. They are important because of their effects on gene expression in the vicinity of the DNA breakpoints where they occur, as well as their direct interruption of some genes. Such inversions are considered to have been major drivers in the speciation process of humans and chimpanzees, and to have contributed greatly to genetic variation between the two species (33). A significant proportion of these inversions, as well as of other chromosomal rearrangements, such as the segmental duplications mentioned earlier, are caused by the actions of the aforementioned mobile (transposable) elements. Together, these elements have acted dynamically to generate numerous, and often substantial, chromosomal rearrangements, which are the basis of many of the genetic differences between humans and chimpanzees, including gene gain and gene loss (33).

In 2005, Feuk and colleagues compared the genomes of the two species and identified almost 1,600 inverted regions, which are equivalent to approximately $5 \%$ of the human genome (39). They noted that 151 of these inversions contained either entire or partial genes, or a breakpoint that intersected a gene. Furthermore, they found 140 genes with human-specific CNDs and 15 genes with chimpanzee-specific CNDs, of which some were important immunologically. A more-recent study identified 252 inversion loci between human and chimpanzee lineages, the junctions of which could be easily characterised. Analysis of these junctions directly implicated the aforementioned LINEs and an abundant class of SINEs, known as Alu elements, in the inversion process (33). This study also revealed a novel mechanism for some inversions that was responsible for 27 human-specific events and for 22 chimpanzee-specific events. These events affected exonic and intronic regions, and had an impact on gene function via gene disruption, the generation of alternative splice sites, and effects on gene regulatory regions. There are approximately one million Alu elements in the human genome with the power to effect these outcomes (40), and thereby greatly influence gene expression (41).

Alu elements can also give rise to completely new exons, often in existing functional genes, with diverse splicing patterns (40). One such Aluderived exon has been implicated in a form of muscular dystrophy (42). More generally, Alu elements have been associated with a number of diseases that arise from disrupted gene function, including various cancers, haemophilia, neurofibromatosis, type-2 diabetes and Alzheimer's disease (43). Interestingly, the chimpanzee genome has 100,000 fewer Alu elements than the human genome, and although 572 evolutionarily young Alu elements have been identified in the human genome, just 160 such elements have been found in the chimpanzee genome (43). These great differences speak once again to the inappropriateness of equating research on chimpanzees to investigations based on human subjects.

Large insertions and deletions of genetic material, or indels, are another source of significant 
variation between human and chimpanzee genomes. Volfovsky and colleagues compared twothirds of human chromosome 21 and its equivalent in chimpanzees, chromosome 22 (there is an alternative chromosome nomenclature, in which human and chimpanzee chromosomes are numbered similarly; based on this alternative nomenclature, the comparison involved human and chimpanzee chromosome 22). This comparison revealed 683 indels in the proximity of known human genes, which potentially affected their expression (44). Twenty-three of these indels were within exons, or within splice-site regions, and ten altered the sequence of the corresponding protein. Indels have also been shown to affect major histocompatibility complex (MHC) genes, which are critical for immune responses. Indeed, indels are responsible for a large $95 \mathrm{~kb}$ deletion in the $\mathrm{MHC}$ locus that resulted in a virtual fusion of the $M I C A$ and $M I C B$ human genes into the single hybrid chimpanzee $M I C$ gene (45). An additional 64 indels of $100 \mathrm{bp}$ were identified in this locus, which is genetically linked to differences in the handling of various infections, including HIV, hepatitis B and $\mathrm{C}$ viruses, and the malarial parasite, Plasmodium falciparum, as well as susceptibility to autoimmune diseases.

\section{Gene complement}

Many genes are present in humans but entirely absent in chimpanzees, or vice versa, largely due to the duplication and deletion of large genomic regions already noted. It has been reported that, since the evolutionary split of humans and chimpanzees, humans have gained 689 genes and lost 86 , while chimpanzees have gained 26 genes and lost 729 that are still present in humans (46). This means that humans differ by $6.4 \%$ in terms of their gene complement alone $(1,418$ genes out of 22,000$)$, which has significant consequences. An example is the golgin gene subfamily, which has been linked to systemic autoimmune diseases such as lupus erythematosus (47). In humans, this subfamily comprises 49 genes, but only 23 genes in chimpanzees.

A comparison of protease genes, which encode enzymes important in a wide variety of cellular processes, showed that five genes had been completely deleted in the human genome, while the corresponding number of deletions in the chimpanzee genome was just two $(48,49)$. Other studies have suggested: that $27 \%$ of human kinase genes might not have a close homologue in chimpanzees (50); that $23 \%$ of human genes encoding zinc-finger proteins, many of which modulate gene transcription, might be absent in chimpanzees (51); and that the presence of genes encoding cellsurface receptors of natural-killer cells, which are implicated in immunity to viral infection and surveillance for cancer, differs between the two species (52).

\section{Gene expression}

Even in the majority of cases in which genes are common to humans and chimpanzees, there are substantial and widespread differences in their expression. For example, a study examining the expression of around 12,000 genes in the prefrontal cortex of the brain found that almost 1,000 were expressed in the human, but not in the chimpanzee, while the reverse occurred for 344 genes. In addition, of the genes that were expressed in both species, $20 \%$ showed a different expression profile - for example, 19 genes linked to Alzheimer's, Parkinson's and Huntington's diseases in humans were found to be expressed differently in chimpanzees (53). In the cerebral cortex, at least 169 genes are expressed differently - many of which are involved in neuroprotection and synaptic transport (54) - and 916 genes are expressed at least two-fold differently in the cerebellum (55). Furthermore, many genes involved in oxidative metabolism and mitochondrial function are expressed to a higher degree in the human brain than in the chimpanzee brain (56-58).

Not surprisingly, such differences are not confined to the brain. One study analysed an average of approximately 10,500 genes in various human and chimpanzee organs, and found that a striking $34 \%$ showed differential expression in the brain, $25 \%$ in the liver, $33 \%$ in the kidney, $35 \%$ in the heart, and $62 \%$ in the testes (59). Another study, which analysed even more genes $(17,231)$, identified $16.3 \%$ of genes in the liver, $19.5 \%$ in the kidney, and $18.5 \%$ in the heart, that were expressed at different levels within the two species (57). These percentages amount to many thousands of genes differentially expressed in various organs. Notably, differences in the expression of a single gene can have crucially important consequences: the natural cytotoxicity receptor gene ( $N K p 44)$, for example, shows five-fold higher expression in chimpanzees than in humans, and it exhibits differential increases upon activation. Its protein product is involved in the recognition of $\mathrm{HIV}-1$, and in the sensing and killing of virus-infected and cancerous cells. It is thought that these differences could be at least partly responsible for the more benign course of HIV infection in chimpanzees (60).

It is highly likely that species variation in the expression of just one gene, which encodes a member of the inhibitory sialic acid-recognising Igsuperfamily lectins (Siglecs), has a major effect on comparative general immune responses to many and varied pathogens. For example, Soto and co- 
workers (61) showed that inhibitory Siglecs generally, but in particular Siglec-5, are expressed at a much lower level in humans than in chimpanzees. The greater abundance of inhibitory Siglecs in chimpanzees serves to dampen excessive immune responses relative to humans, leaving human $\mathrm{T}$ lymphocytes and B-lymphocytes, in comparison to those of chimpanzees, over reactive to a variety of stimuli. This may explain species differences in diseases that involve immunopathology, including HIV, hepatitis C, asthma, psoriasis and rheumatoid arthritis (61).

In addition, terminal sialic acids are produced in larger amounts in the hearts of chimpanzees than they are in those of humans (62). As these molecules are frequently the targets of viral pathogens that can initiate fibrosis, their greater abundance in chimpanzees makes myocardial fibrosis more common in that species (63). Indeed, this type of heart disease is the major cause of cardiac arrest and progressive heart failure in chimpanzees, but it is very different from the main cause of heart disease in humans, which is coronary artery atherosclerosis (63). In common with the apparent genetic factors underlying a greater incidence of myocardial fibrosis in chimpanzees, it seems that the increased incidence of atherosclerosis in humans might also have a genetic cause - the lack of Neu5Gc sialic acid (described further in the Differences in Genetic Sequences section). A theory is that humans ingest Neu5Gc sialic acid (as part of their diet), and that this 'foreign' molecule triggers an immune response, causing inflammation and exacerbating atherosclerosis. This chain of events is augmented by the increased reactivity of human T-cells caused by their lower expression of inhibitory Siglecs, as noted above (61). In summary, it appears that a few differences in genetic sequence and gene expression may be responsible for very different pathologies of heart disease in humans and chimpanzees (63).

\section{Factors affecting gene expression}

It is significant that many of the genes that are differentially expressed, often at a higher level in humans, encode transcription factors, as these proteins can affect the regulation of many hundreds of genes and can result in significant phenotypic effects (64). Despite their understandably strict evolutionary conservation, many transcription factors have evolved under directional selection in humans $(57,65)$. A study by Nowick et al. illustrates this elegantly, as they identified 90 transcription factor genes with significantly different expression levels in human and chimpanzee brains (66). These gene networks were enriched for primate-specific $K R A B-Z N F$ genes, which are central to human and chimpanzee brains and are associ- ated with genes involved in the development and maintenance of this organ.

Species differences in transcription factors create variations in gene expression, and thus generate physiological changes between humans and chimpanzees. In addition, the role of transcription factors in differential gene expression is augmented by variations in transcription factor binding sites between species. DNA sequence variability at transcription factor binding sites is normal between individuals of the same species: for example, $25 \%$ of binding sites for RNA polymerase II vary between individual humans. However, a statistically significant $32 \%$ of these binding sites were found to have meaningful differences between humans and chimpanzees (67). Differences in conserved non-coding sequences flanking genes have been shown to have serious implications for gene expression, as they may affect three-quarters of human autosomal genes (75.4\% in one particular study; 68).

As an example of species-specific variability in the influence of neighbouring non-coding regions, a comparative analysis of 100 representative DNA repair-associated genes in humans and chimpanzees revealed that $13 \%$ and $32 \%$, respectively, exhibited evidence of accelerated evolution in their promoter and intronic regions (69). Some of those genes also showed inter-species differences in expression, which are thought to affect DNA repair mechanisms. A faster rate of DNA repair in chimpanzee cells than in human cells, might contribute to the different incidences of cancer between the species.

Gene expression is also affected by DNA methylation patterns, which act epigenetically through histone modification and chromatin remodelling to mediate transcription (70). In common with all alterations to cis-acting sequences, minor variations, or differences, in DNA methylation can affect the expression of many genes. It has been discovered that over $12 \%$ of methylation sites, covering one-third of all genes, might be differentially methylated in humans and chimpanzees (71).

There are major differences in other factors that control gene expression. For example, in recent years microRNAs - small, 21-22 nucleotide RNA molecules that can negatively regulate gene expression by interfering with mRNA molecules, either causing them to degrade or by blocking transcription - have received increasing attention (72, 73). There are thought to be over 1,000 miRNAs, each of which can repress the expression of hundreds of genes in highly complex regulatory networks (74). They play crucial roles in cell development, e.g. in signalling and differentiation, and in cell fate, e.g. in apoptosis. As miRNAs regulate many oncogenes, perturbation of their function can induce the initiation and progression of tumours (75). Interestingly, comparisons of human 
and chimpanzee precursor miRNAs have shown that only $60 \%$ are identical, and that just $73 \%$ show no change in their secondary structure (76). In addition, around $10 \%$ of mature miRNAs are different to some degree, and there are at least 39 miRNAs that are present in the human but not in the chimpanzee (75). As a result of the fast evolution of miRNA binding sites (76), there are undoubtedly species differences in the expression levels of miRNAs (and in the selection and expression of target genes). At least $10 \%$ of miRNAs in embryonic stem cells, which can differentiate into over 200 different types of cell during development, differ between humans and chimpanzees (77).

Adenosine-to-inosine RNA editing, another important factor that affects gene expression, is catalysed by an enzyme that post-transcriptionally converts adenosine to inosine in RNA molecules, resulting in RNA sequences in which inosines, in place of the genomically-encoded adenosines, are read as guanosine residues by the cell's translational and splicing machinery. This process has obvious consequences for any gene that is affected, but importantly the process of editing is considerably more prominent in humans than in chimpanzees (78). In humans, it occurs predominantly in the previously described Alu elements, and it affects myriad loci across tens of thousands of genes. When Alu elements in non-coding regions are edited, gene expression can be altered; when coding regions are edited, the amino acid sequences, properties and functions of the encoded proteins are changed. Because the brain is most affected in this way, many of the targets of editing are associated with the genes involved in neurogenesis. Altered editing is therefore associated with a number of neuropathological disorders. The differential editing between humans and chimpanzees has distinct effects on gene expression and function via alternative splicing and differences in mRNA stability, nuclear retention and miRNA biogenesis and targeting (78).

\section{Differences in genetic sequences}

In addition to the large-scale genomic rearrangements, such as inversions, indels, duplications, and so forth already discussed, as well as the disparities in gene complement, there are smaller apparent differences that can have a considerable impact on human and chimpanzee physiology and that are responsible for notable inter-species differences.

A recent analysis of chimpanzee genes 'equivalent' to 333 human genes implicated in cancer (79) found that the genes shared a high degree of similarity. This would be expected, because genes controlling cell growth are often tightly conserved throughout evolution. However, 20 of the 333 genes, some of which are definitively involved in tumour formation, had some level of difference. This may help to explain why chimpanzees have an extraordinarily low incidence of cancers, especially those of the breast, prostate and lung, cancers that account for over $20 \%$ of human deaths in modern populations (12). Another analysis, this one of human and chimpanzee protease genes (which are involved in many essential biological processes), revealed important differences: a high degree of similarity was discovered between the 559 chimpanzee and 561 human protease genes, but seven genes were deleted entirely (i.e. two genes present in chimpanzees were absent in humans, and the opposite was true for five genes; 48). Many other genes contained small insertions, deletions or sequence changes that can lead to gene inactivation, and several of these genes were involved in the function of the immune system (48).

Another study identified 54 genes in humans and 162 genes in chimpanzees that had undergone positive selection since the evolutionary split between the species. Just 17 genes had been positively selected in both species (80). Notably, the genes positively selected in humans have been implicated in epithelial cancers, schizophrenia and other cognitive disorders, ataxia and migraine, autoimmune diseases such as lupus and rheumatoid arthritis, and Alzheimer's disease, all of which differ in prevalence and symptoms between humans and chimpanzees (80). The positively selected genes included those involved in: DNA repair, general tumour progression, gastric and breast cancers, susceptibility to and progression of melanoma, neurological development, various ataxias, migraine, dystonia, epilepsy, bipolar disorder and cognitive impairment. Differences in transcription factor genes were also found, including the HIVEP3 gene, which activates gene expression in HIV and which may account for some of the species differences in HIV/AIDS, and other genes involved in regulating gene expression such as MOV10, which is involved in the silencing of mRNA molecules.

Although Volfovsky et al. (44), in the study previously reported, identified 683 large indels between chimpanzee chromosome 22 and its human counterpart chromosome 21, an earlier, more-detailed analysis of these chromosomes revealed a total of around 68,000 indels of all sizes, resulting in genetic differences in the 231 genes therein, leading to differences between humans and chimpanzees in $83 \%$ of the protein products (81). In addition, around 1 in 10 of these genes showed significant differences in their expression levels. Another study found that about $8 \%$ $(1,109 / 13,487)$ of the human/chimpanzee gene pairs analysed were affected by premature stop 
codons in the chimpanzee version, resulting in truncated mRNA transcriptional products that are either degraded, or that give rise to truncated proteins with altered or ablated functions (17). Elsewhere, an analysis of all human and chimpanzee exons showed that humans have 1,931 disrupted exons (there are more than 500,000 in total), while the chimpanzee has 3,742 disrupted exons (82). In humans, functional consequences of these disruptions identified to date include melanoma, breast cancer, neuroblastoma, some inflammatory diseases, problems of immune and cardiovascular development, lupus and stress responses.

Specific examples of small genetic changes with major, or at least potentially major, consequences are commonplace. For example, the CMP-sialic acid hydroxylase gene in humans contains a small deletion of a $92 \mathrm{bp}$ exon that is not found in the chimpanzee gene. This deletion causes a frameshift that results in loss of function of the protein. Deficiency of this enzyme activity means that humans, unlike chimpanzees, do not have a particular sialic acid residue ( $N$-glycolyl-neuraminic acid, or Neu5Gc) on the surface of almost all of the cells in their bodies. The absence of this residue has implications for intercellular interactions and many other biological processes, as well as for susceptibility (and resistance) to microbial pathogens (83). The CDR4 gene, which encodes the CDR4 cell receptor that is a major part of the receptor complex for HIV and thus its route of infection of immune cells, differs between humans and chimpanzees significantly. This difference affects CDR4 regulation, expression, density on the cell surface and glycosylation - factors that influence its structure, stability, and ability to interact with other immune cells. These small genetic differences between humans and chimpanzees therefore have serious consequences for HIV infection and pathology, and underlie some of the differences seen in HIV/AIDS between the species (84).

The differences in the genomic complement of protease genes (48), which were discussed earlier, may be significant, despite the overall similarity of the human and chimpanzee degradomes and their constituent genes. Important differences also exist in protease genes that are common to the two species, particularly in those genes related to host defence, such as neutrophil granule proteases and processors of pro-inflammatory cytokines (49). These differences are notable, because of the wide variety of critical functions in which the enzymes are involved, such as: cell cycle progression; cellular proliferation, differentiation and migration; embryonic development; tissue remodelling; angiogenesis; apoptosis, autophagy and senescence; fertilisation; immunity; wound healing; and haemostasis (49). The importance of proteases, and of the effects of their differential regulation or mutation, is further reflected in the array of human diseases with which they are associated, including: cancer, arthritis, neurodegenerative and cardiovascular disorders, haemophilia, Alzheimer's disease, hereditary pancreatitis, and progeroid syndromes (49).

A deletion of two base pairs in the human MYH16 gene resulted in a frameshift that reduced the sizes of jaw muscles in humans and allowed a larger brain to develop (85). A single base pair substitution caused an inactivating, premature stop codon in the human type I hair keratin gene, which resulted in smooth, hairless skin in humans, in contrast to the very hairy skin of chimpanzees (86). Sequence differences in the FOXP2 gene, resulting in just two amino acids that are different in the protein product, have been linked with a species difference of almost immeasurable magnitude and consequence - the acquisition of human language (87).

While inter-species sequence disparities for any particular gene may have obvious direct consequences due to the potential variability of that gene's protein products and their immediate functional activities, it must also be appreciated that indirect effects may be significant as well. The aforementioned FOXP2 gene, for example, in addition to its own structural and functional differences between humans and chimpanzees, differentially and significantly affects the expression of a further 116 genes. In humans, but not in chimpanzees, 61 genes are up-regulated and another 55 are down-regulated by the FOXP2 protein (88). The genes involved are important for brain development and function (for example, those involved in craniofacial formation and in establishing the neural circuitry and physical structures needed for spoken language via cerebellar motor function), and in the formation of cartilage and connective tissue.

\section{Differences in RNA splicing}

Splicing factors are complexes of proteins and RNA that bind to specific sequences in pre-mRNA intermediates during transcription, and splice out intronic sequences to generate multiple mRNA molecules from one gene. Their presence, nature, function and repertoire, therefore have far-reaching consequences for the expression of many other genes. They greatly increase the complexity of the genome and proteome, and over $80 \%$ of human genes are subject to their action (89). It is critical to note that many splicing factors are differentially expressed in humans and chimpanzees; for example, 43 in the testes and 20 in the brain (90). This alone will result in many protein variants, which may have distinct functions in the tissues and organs of humans and chimpanzees. As previously 
described, the inter-species consequences of differential alternative splicing are further amplified by the differential action of mobile elements and adenosine-inosine RNA editing in generating and deleting alternative splice sites $(33,40,44,78)$.

\section{Post-translational differences}

The genomic and transcriptomic differences described earlier obviously translate into proteomic differences, with consequent inter-species diversity in protein function, biochemistry and physiology. In addition, there are comparative proteomic studies that directly inform our knowledge of human/chimpanzee differences at the protein level. For example, $80 \%$ of orthologous proteins were found to differ in their amino acid sequences (91) by an average of two amino acids per protein (92), which has clear implications for evaluating the applicability of data from chimpanzees. Furthermore, a comparative analysis of proteins that interact with HIV-1 revealed that, of 1,447 such human proteins, 77 had no orthologue in nonhumans, including chimpanzees (93). The same study revealed significant differences between humans and chimpanzees in the group of APOBEC3 proteins, which are involved in host defence against retroviruses. Of the 1,370 human and chimpanzee orthologous proteins that were compared, each species had more than 600 speciesspecific phosphorylation sites, potentially affecting thousands of protein-protein interactions, enzyme activities and protein stabilities (93).

\section{The consequences of genetic differences: Evaluation of the translation of data from chimpanzee research to humans}

Several critical analyses have reflected serious concerns about the human relevance of chimpanzee research and the capacity of chimpanzee data to produce tangible clinical benefits. While each of these representative studies set out with the hypothesis that chimpanzee experimentation was not a productive, or necessary, research methodology, their conclusions are supported by substantial data. Furthermore, it must be noted that each study provides numerous peer-reviewed publications, as well as professional opinion and comment to substantiate its conclusions. Caveats surrounding the efficacy of chimpanzee research are indeed widespread, and they emanate from many and varied qualified sources.

In 2007, a citation analysis by Knight (13) and Bailey and colleagues (see http://www.release chimps.org/pdfs/chimp-efficacy-paper-main.pdf and http://www.releasechimps.org/pdfs/Chimp-CAsuppl.pdf) provided a general overview of the per- ceived and actual importance of chimpanzee research, amid claims from its advocates that it is crucial and takes place only when absolutely necessary (as expressed, for example, by VandeBerg and Zola [4]). If these claims were actually wellfounded, papers reporting chimpanzee research should be heavily cited in the literature describing advances in human medicine, but the 2007 analysis found this not to be the case. By using a statistically representative sample of almost 100 published papers, the analysis revealed that more than $85 \%$ of the chimpanzee studies had not been cited at all in papers relevant to human medicine. Furthermore, a thorough and detailed analysis of the papers cited in a human medical context, and of those that had cited them, revealed that the chimpanzee studies had contributed little, if anything, to the outcome of the human studies. Often, the results from research involving chimpanzees were duplications of human outcomes that had already been reported in earlier papers, or the conclusions were inconsistent with data on humans or other non-human primates. In contrast, the analysis found it demonstrable that in vitro research, human clinical and epidemiological investigations, and molecular and genomic methods had contributed most to the findings of the 95 papers in the sample.

A review that examined the use of chimpanzees in HIV/AIDS research (11) found that the number of AIDS-related chimpanzee studies fell by nearly $90 \%$ between 1998 and 2005, apparently because of the poor clinical translation of, and need for, that line of research. Responses to HIV vaccines were highly discordant in humans and chimpanzees, so the review concluded that responses to vaccination in chimpanzees could not be considered predictive of responses in humans. In fact, according to the same review, 85 diverse HIV vaccines that were developed by using chimpanzees and other primates and shown to have protective, or therapeutic effects, were evaluated in 197 human clinical trials. Yet, protection and/or significant therapeutic effects in humans have still not been demonstrated by any HIV vaccine, despite decades of effort and many millions of dollars of research funding. Thus, contrary to claims that chimpanzees "are still important for testing vaccines aimed at preventing HIV-1 infection or reducing the virus load in infected individuals", for example (4), it was concluded that neither claim has any scientific foundation, and that a return to the use of chimpanzees in AIDS research/vaccine development would be without scientific justification.

Chimpanzees have scarcely been used in any form of cancer research, which may be surprising when one considers that cancer is one of the greatest killers of human beings. A review of the subject showed that there have been very few scientific publications relevant to human cancer that used 
chimpanzees, and that tumours in chimpanzees are extremely rare, especially of the types that are responsible for most of the deaths from cancer among humans (12). The review found that chimpanzee tumours are biologically different from those of humans, and this includes the tumour initiation and progression phases. These observations demonstrate that, despite their overall apparent genetic similarity to humans, chimpanzees constitute a poor research model for human cancer. Furthermore, these animals are not essential for the development of therapeutic monoclonal antibodies for cancer treatment. Finally, the review found that published papers describing potential new cancer therapies tested in chimpanzees often included significant caveats concerning differences in the species, acknowledged that research with chimpanzees was no better than research with other animals, and described interventions that had not been pursued clinically, presumably due to adverse preclinical results (12). These conclusions are supported by many of the genetic differences between humans and chimpanzees described in the present paper.

Finally, two in-depth reviews by the author of the present paper have recently been completed. These address claims that research with chimpanzees must continue in order to provide a better understanding of hepatitis $\mathrm{C}$, and to realise treatments and cures for this devastating disease that affects millions of people worldwide $(9,10)$. This is important, not only due to the impact of hepatitis $\mathrm{C}$ on the human population, but also because it represents the area of greatest use of chimpanzees in laboratories at present. These two reviews demonstrate that researchers have, for many years, cited numerous serious caveats and problems with the chimpanzee model. They have also stressed the urgent need for in vitro viral culture systems to accelerate progress, which occurred much earlier for other viruses, such as polio and measles. Paralleling the case of HIV/AIDS, these caveats, and the need for in vitro culture systems, are due to major pathological differences between human and chimpanzee hepatitis $\mathrm{C}$ virus infection. The reviews show that in vitro and clinical approaches, rather than chimpanzee experiments, have provided the most substantial, comprehensive and important data. It is now possible to investigate the entire life cycle of the hepatitis $\mathrm{C}$ virus, immune responses and host factors, identify therapeutic targets, and test new therapies and vaccines, in a human, and therefore completely relevant, context. The reviews show that chimpanzee use is declining markedly in research on hepatitis $\mathrm{C}$; indeed, it has fallen by over $60 \%$ in the last 20 years and is now at a historical low of just onethird of its 1985 peak level. In contrast, non-animal research on hepatitis $\mathrm{C}$ has increased 80 -fold over the same time period. In summary, in the area of hepatitis $\mathrm{C}$ research, the arguments are strongly against a scientifically based requirement for the use of chimpanzees and very much in favour of concentrating research in human-specific clinical and in vitro technologies.

\section{Discussion and Conclusions}

The conduct of invasive research on captive chimpanzees may never have been more controversial than it is today. In recent years, experimentation on chimpanzees has been outlawed, or at least severely restricted, in the relatively few scientifically advanced nations where it was ever practised (see http://www.releasechimps.org/mission/end-chimpanzee-research/country-bans/). Nevertheless, some observers still insist that such restriction is a mistake (4). Legislation banning experimentation on chimpanzees has been implemented largely due to ethical concerns - namely, that research with chimpanzees causes extreme pain and suffering to animals with very highly developed cognitive and emotional capacities. This rationale is being increasingly augmented by a burgeoning scientific argument - that data from chimpanzee experiments are flawed and invalid with regard to human medicine, and that superior alternatives, with greater human relevance, do exist. This argument, in turn, is bolstered by our increasing knowledge of the genetic differences between humans and chimpanzees, as was summarised in this review, which serve to explain the negative results and conclusions reached in critical assessments of the relevance of the chimpanzee for many areas of research on human disease. These negative results and conclusions, briefly summarised above, also give value and meaning to those genetic differences, showing that they do indeed matter and that they combine to make the chimpanzee a very different species from the human.

Yet, in spite of this substantial and compelling knowledge, the ostensibly extreme genetic similarity of chimpanzees and humans is frequently used to assert the validity and indispensability of chimpanzee research. An example from 2005 is a highprofile opinion piece by two prominent chimpanzee researchers, from two institutions housing hundreds of research chimpanzees, in which they make a plea for increased research on captive chimpanzees (4). It is conceded that the chimpanzee is the species most genetically similar to humans, although the complexity of aligning such huge genomes makes it difficult, if not impossible, to measure this similarity in a truly precise way. Initial estimates suggested this similarity was around $98.5 \%$ to $99 \%$ (King and Wilson [94], reviewed by Varki [95]), a figure that has been confirmed more recently, although with certain caveats (reviewed by Volfovsky et al. [44], 
Watanabe et al. [81], and the Chimpanzee Sequencing and Analysis Consortium [92], among others). For instance, the figure of 98.5-99\% takes into account nucleotide substitutions only. When genomic insertions and deletions are considered, the overall similarity decreases to approximately 95-96\% (14, 15), or to as low as 93.5\% (16). Even when repeat-sequence and low-complexity DNA are excluded, the overall difference is still thought to be approaching $2.5 \%$, which is double the original and highly cited estimate (16).

It has been widely acknowledged for some years that a genetic difference of only $1-2 \%$ is actually a 'myth', but its fragile underpinnings have apparently been overlooked by advocates of chimpanzee research in their defence of the practice. Their assertion of a high genetic similarity and of only a $1-2 \%$ difference is even more surprising when one appreciates the extent of the awareness within the scientific community of other important interspecies disparities and their phenotypic effects, and for how long this awareness has existed. As long ago as 1975, the authors of a seminal study (94) that indicated a 1\% difference between humans and chimpanzees insisted that "the genetic distance between humans and the chimpanzee is probably too small to account for their substantial organismal differences". In other words, differences in gene regulation and expression, rather than protein-coding mutations, must play a major role $(46,94,96)$. Knowledge of other factors with a great influence on gene expression and human/chimpanzee phenotypes also precedes and surrounds such claims. As this review demonstrates, various phenomena - including genomic rearrangements, mobile DNA elements (e.g. LINEs and SINEs), gene duplications and deletions, copy number variation, differences in transcription factors and binding sites, DNA methylation, miRNAs and binding sites, gene editing and splicing and protein phosphorylation contribute to human/chimpanzee biological differences. Many of these factors affect gene expression and function, and are deemed to have played a greater role than simple nucleotide substitutions in the evolution of species-specific phenotypes. As opined in a review outlining significant gene gain and loss in humans and chimpanzees: "Without accounting for differences in the total DNA unique to each species, we cannot hope to take a proper accounting of the meaningful genetic divergence between humans and chimpanzees" (46).

As the recent analyses summarised in this review show, the divergence between humans and chimpanzees is considerable, and it translates to major differences in susceptibility to disease, in symptoms and in pathologies. These differences have "eluded any molecular explanation within this supposedly 1\% diversity range" (45), a diversity which "is clearly misleading" (91). Humans, for example, are more susceptible to a variety of "infectious diseases, cardiovascular diseases, obesity, type II diabetes, autoimmune diseases, major psychoses, and neurodegenerative diseases" (69), are more prone to developing cancer $(12,59)$, and are differently susceptible to malarial parasites when they are compared to chimpanzees (97). Experimentation with chimpanzees has failed to translate to meaningful progress in HIV/AIDS (11), hepatitis C $(9,10)$ and cancer $(12)$, as well as more generally for other diseases (13; see also http://www.releasechimps.org/pdfs/chimp-efficacypaper-main.pdf and http://www.releasechimps. org/pdfs/Chimp-CA-suppl.pdf).

The use of chimpanzees has declined greatly in all areas of research, and it cannot be shown to be necessary in any current field of biomedical research. It has been falsely credited with major breakthroughs in the past, where human-specific methods were the true contributors, while modern technologies and cutting-edge alternative methods negate any value it may have had historically. The presumed benefits are arguable in themselves. It must therefore be concluded that any justification of chimpanzee research based on genetic similarity is superficial and incorrect. And, based on a deeper and more thorough appreciation of profound interspecies differences in gene expression, rather than simple genetic similarity, it must be concluded that the chimpanzee does not, and can never, constitute a crucial and indispensable model for human biomedical research.

When the scientific case against chimpanzee experimentation is considered alongside the numerous and varied concerns about animal welfare, ethical issues and financial concerns $(7,8)$, the argument becomes even more compelling and formidable. Studies have revealed post-traumatic stress disorder in ex-research chimpanzees now in sanctuary $(5,98)$, and have detailed physical and psychological trauma suffered by chimpanzees that have been raised in various human/chimpanzee contexts and then used in research, as well as the chimpanzees' ability to recover from such trauma once in sanctuary (6). Tens of millions of taxpayer dollars could be saved each year by transferring all federally owned and supported chimpanzees from laboratories to sanctuaries, where they would receive superior care and enjoy a higher quality of life (Capaldo and Owens, submitted). Public opinion polls show that twice as many Americans support a ban on chimpanzee research as oppose one, and that $71 \%$ of the American public think chimpanzees used in research for more than 10 years should be retired (this figure would now include over $90 \%$ of all chimpanzees in US laboratories; see http://www.releasechimps.org/mission/end-chimpanzee-research/public-opinion/). Finally, chimpanzee experimentation is a 'special case'. This is evidenced not only by public opinion, 
but also by various policies and laws in the USA, such as: the CHIMP Act passed in 2000 (99); the extension, in 2007, of a moratorium in place since 1995 on breeding chimpanzees which applies to federally supported chimpanzees in US laboratories (see http://www.releasechimps.org/2007/05/ 22/breeding-moratorium-decision-good-news/); the many countries that have recently banned or severely limited the use of great apes (see http:// www.releasechimps.org/mission/end-chimpanzeeresearch/country-bans/); and the considerable legislative support in both the US House of Representatives and the US Senate for GAPCSA (the Great Ape Protection and Cost Savings Act), which seeks to end invasive chimpanzee research and retire all federally owned chimpanzees in US laboratories to permanent sanctuary $(23,24)$.

To the best available knowledge, the USA is the only country in the world that actively conducts invasive research on captive chimpanzees to any significant degree. For the sake of the approximately 1,000 chimpanzees in US laboratories, who almost without exception are elderly and have suffered greatly, there is a strong argument for the USA to join the rest of the world and end this research. There is also a strong argument to end this research for the sake of humanity, which stands to benefit from superior, more humane, and more human-relevant scientific inquiry, should the use of chimpanzees cease. The evidence reviewed here shows that chimpanzees are simply not 'human enough' to constitute valuable models for research on human diseases: important differences, with serious biological consequences, run right through each level of gene expression and protein function, from chromosome and chromatin structure to post-translational modification. This results in non-predictive data of poor relevance to humans, that rarely translate to the clinic and that actually impede progress in human medicine. The argument that chimpanzees must constitute a good model for research on human diseases, based on their ostensible genetic similarity to humans, is specious and should be dismissed. Only by moving away from chimpanzee research, and by fully embracing and adopting superior human-specific alternatives, can treatments or cures for the many diseases that blight the lives of hundreds of millions of people be realised quickly and safely.

\section{Acknowledgements}

Jarrod Bailey was the sole author of this manuscript and was responsible for its conception, research, and preparation. The work herein has not been presented anywhere else in print prior to this publication, and is based on an oral presentation given at the 'Animals, Research and Alternatives' conference in Washington, DC, USA, on 26 August, 2010. Sincere gratitude is expressed to the New England Anti-Vivisection Society (NEAVS) and the British Union for the Abolition of Vivisection (BUAV) for funding this work. There are no financial conflicts of interest. The author is supported by these two not-for-profit groups, which campaign against animal experimentation.

Received 30.06.11; received in final form 14.09.11; accepted for publication 14.09.11.

\section{References}

1. Kohne, D.E. (1970). Evolution of higher-organism DNA. Quarterly Reviews of Biophysics 3, 327-375.

2. Sibley, C.G. \& Ahlquist, J.E. (1984). The phylogeny of the hominoid primates, as indicated by DNA-DNA hybridization. Journal of Molecular Evolution 20, 2-15.

3. Ebersberger, I., Metzler, D., Schwarz, C. \& Pääbo, S. (2002). Genomewide comparison of DNA sequences between humans and chimpanzees. American Journal of Human Genetics 70, 1490-1497.

4. VandeBerg, J.L. \& Zola, S.M. (2005). A unique biomedical resource at risk. Nature, London 437, 30-32.

5. Bradshaw, G.A., Capaldo, T., Lindner, L. \& Grow, G. (2008). Building an inner sanctuary: Complex PTSD in chimpanzees. Journal of Trauma \& Dissociation 9, 9-34.

6. Bradshaw, G.A., Capaldo, T., Lindner, L. \& Grow, G. (2009). Developmental context effects on bi-cultural post-trauma self repair in chimpanzees. Developmental Psychology 45, 1376-1388.

7. Sauer, U.G. (2000). Reasons for not using primates in research. ALTEX 17, 217-220. [In German.]

8. Thew, M. (2002). Are results of primate research worth the suffering it causes? Nature, London 418, 273.

9. Bailey, J. (2010). An assessment of the use of chimpanzees in hepatitis C research past, present and future: 1 . Validity of the chimpanzee model. ATLA 38, 387-418.

10. Bailey, J. (2010). An assessment of the use of chimpanzees in hepatitis $C$ research past, present and future: 2. Alternative replacement methods. ATLA 38, 471-494.

11. Bailey, J. (2008). An assessment of the role of chimpanzees in AIDS vaccine research. ATLA $\mathbf{3 6}$, 381-428.

12. Bailey, J. (2009). An examination of chimpanzee use in human cancer research. ATLA 37, 399-416.

13. Knight, A. (2007). The poor contribution of chimpanzee experiments to biomedical progress. Journal of Applied Animal Welfare Science 10, 281-308.

14. Britten, R.J. (2002). Divergence between samples of chimpanzee and human DNA sequences is 5\%, counting indels. Proceedings of the National Academy of Sciences of the USA 99, 13,633-13,635.

15. Varki, A. \& Altheide, T.K. (2005). Comparing the human and chimpanzee genomes: Searching for needles in a haystack. Genome Research 15, 1746-1758.

16. Wetterbom, A., Sevov, M., Cavelier, L. \& Bergstrom, T.F. (2006). Comparative genomic analysis of human and chimpanzee indicates a key role for 
indels in primate evolution. Journal of Molecular Evolution 63, 682-690.

17. Wetterbom, A., Gyllensten, U., Cavelier, L. \& Bergstrom, T.F. (2009). Genome-wide analysis of chimpanzee genes with premature termination codons. BMC Genomics 10, 56.

18. Institute for Laboratory Animal Research US (1997). Committee on Long-term Care of Chimpanzees. Chimpanzees in Research: Strategies for Their Ethical Care, Management, and Use (Compass Series), 108pp. Washington, DC, USA: National Academies Press.

19. Shalev, M. (2001). President Clinton signs the Chimpanzee Health, Improvement, Maintenance, and Protection Act. Lab Animal 30, 15-16.

20. Vogel, G. (2000). Laboratory animals: Congress OKs plan for retired chimps. Science 290, 2233a.

21. OpenCongress (2009). H.R.1326 - Great Ape Protection Act. New York, NY, USA: OpenCongress. Available at: http://www.opencongress.org/bill/111h1326/show (Accessed 03.10.11).

22. OpenCongress (2010). S.3694 - Great Ape Protection Act. New York, NY, USA: OpenCongress. Available at: http://www.opencongress.org/bill/111s3694/show (Accessed 03.10.11).

23. The Library of Congress (2011). S.810 - Great Ape Protection and Cost Savings Act of 2011. Washington, DC, USA: The Library of Congress. Available at: http://thomas.gov/cgi-bin/query/z?c112:S.810: (Accessed 03.10.2011).

24. The Library of Congress (2011). H.R.1513 - Great Ape Protection and Cost Savings Act of 2011. Washington, DC, USA: The Library of Congress. Available at: http://thomas.gov/cgi-bin/query/z?c112: H.R.1513: (Accessed 03.10.11).

25. Technische Universität Dresden (2009). GoPub Med. Dresden, Germany: Transinsight GmbH. Available at: http://www.gopubmed.com/ (Accessed 03.10.11).

26. Gagneux, P. \& Varki, A. (2001). Genetic differences between humans and great apes. Molecular Phylogenetics \& Evolution 18, 2-13.

27. Kehrer-Sawatzki, H. \& Cooper, D.N. (2007). Understanding the recent evolution of the human genome: Insights from human-chimpanzee genome comparisons. Human Mutation 28, 99-130.

28. Yunis, J.J. \& Prakash, O. (1982). The origin of man: A chromosomal pictorial legacy. Science 215, 1525-1530.

29. Kleinjan, D.J. \& van Heyningen, V. (1998). Position effect in human genetic disease. Human Molecular Genetics 7, 1611-1618.

30. De, S., Teichmann, S.A. \& Babu, M.M. (2009). The impact of genomic neighborhood on the evolution of human and chimpanzee transcriptome. Genome Research 19, 785-794.

31. Hughes, J.F., Skaletsky, H., Pyntikova, T., Graves, T.A., van Daalen, S.K., Minx, P.J., Fulton, R.S., McGrath, S.D., Locke, D.P., Friedman, C., Trask, B.J., Mardis, E.R., Warren, W.C., Repping, S., Rozen, S., Wilson, R.K. \& Page, D.C. (2010). Chimpanzee and human Y chromosomes are remarkably divergent in structure and gene content. Nature, London 463, 536-539.

32. Deininger, P.L., Moran, J.V., Batzer, M.A. \& Kazazian, H.H.J. (2003). Mobile elements and mammalian genome evolution. Current Opinion in Genetics \& Development 13, 651-658.

33. Lee, J., Han, K., Meyer, T.J., Kim, H.S. \& Batzer,
M.A. (2008). Chromosomal inversions between human and chimpanzee lineages caused by retrotransposons. PLoS One 3, e4047.

34. Cheng, Z., Ventura, M., She, X., Khaitovich, P., Graves, T., Osoegawa, K., Church, D., DeJong, P., Wilson, R.K., Pääbo, S., Rocchi, M. \& Eichler, E.E. (2005). A genome-wide comparison of recent chimpanzee and human segmental duplications. Nature, London 437, 88-93.

35. Bailey, J.A. \& Eichler, E.E. (2006). Primate segmental duplications: Crucibles of evolution, diversity and disease. Nature Reviews Genetics 7, $552-564$.

36. Armengol, G., Knuutila, S., Lozano, J.J., Madrigal, I. \& Caballin, M.R. (2010). Identification of human specific gene duplications relative to other primates by array CGH and quantitative PCR. Genomics 95, 203-209.

37. Perry, G.H., Yang, F., Marques-Bonet, T., Murphy, C., Fitzgerald, T., Lee, A.S., Hyland, C., Stone, A.C., Hurles, M.E., Tyler-Smith, C., Eichler, E.E., Carter, N.P., Lee, C. \& Redon, R. (2008). Copy number variation and evolution in humans and chimpanzees. Genome Research 18, 1698-1710.

38. Munch, C., Kirsch, S., Fernandes, A.M. \& Schempp, W. (2008). Evolutionary analysis of the highly dynamic CHEK2 duplicon in anthropoids. BMC Evolutionary Biology 8, 269.

39. Feuk, L., MacDonald, J.R., Tang, T., Carson, A.R., Li, M., Rao, G., Khaja, R. \& Scherer, S.W. (2005). Discovery of human inversion polymorphisms by comparative analysis of human and chimpanzee DNA sequence assemblies. PLoS Genetics 1, e56.

40. Lin, L., Shen, S., Tye, A., Cai, J.J., Jiang, P., Davidson, B.L. \& Xing, Y. (2008). Diverse splicing patterns of exonized Alu elements in human tissues. PLoS Genetics 4, e1000225.

41. Hasler, J. \& Strub, K. (2006). Alu elements as regulators of gene expression. Nucleic Acids Research 34, 5491-5497.

42. Moghadaszadeh, B., Petit, N., Jaillard, C., Brockington, M., Roy, S.Q., Merlini, L., Romero, N., Estournet, B., Desguerre, I., Chaigne, D., Muntoni, F., Topaloglu, H. \& Guicheney, P. (2001). Mutations in SEPN1 cause congenital muscular dystrophy with spinal rigidity and restrictive respiratory syndrome. Nature Genetics 29, 17-18.

43. Srikanta, D., Sen, S.K., Huang, C.T., Conlin, E.M., Rhodes, R.M. \& Batzer, M.A. (2009). An alternative pathway for Alu retrotransposition suggests a role in DNA double-strand break repair. Genomics 93, 205-212.

44. Volfovsky, N., Oleksyk, T.K., Cruz, K.C., Truelove, A.L., Stephens, R.M. \& Smith, M.W. (2009). Genome and gene alterations by insertions and deletions in the evolution of human and chimpanzee chromosome 22. BMC Genomics 10, 51.

45. Anzai, T., Shiina, T., Kimura, N., Yanagiya, K., Kohara, S., Shigenari, A., Yamagata, T., Kulski, J.K., Naruse, T.K., Fujimori, Y., Fukuzumi, Y., Yamazaki, M., Tashiro, H., Iwamoto, C., Umehara, Y., Imanishi, T., Meyer, A., Ikeo, K., Gojobori, T., Bahram, S. \& Inoko, H. (2003). Comparative sequencing of human and chimpanzee MHC class I regions unveils insertions/deletions as the major path to genomic divergence. Proceedings of the National Academy of Sciences of the USA 100,7708-7713.

46. Demuth, J.P., De Bie, T., Stajich, J.E., Cristianini, N. \& Hahn, M.W. (2006). The evolution of mam- 
malian gene families. PLoS One 1, e85.

47. Eystathioy, T., Jakymiw, A., Fujita, D.J., Fritzler, M.J. \& Chan, E.K. (2000). Human autoantibodies to a novel Golgi protein golgin-67: High similarity with golgin-95/gm130 autoantigen. Journal of Autoimmunity 14, 179-187.

48. Puente, X.S., Gutierrez-Fernandez, A., Ordonez, G.R., Hillier, L.W. \& Lopez-Otin, C. (2005). Comparative genomic analysis of human and chimpanzee proteases. Genomics 86, 638-647.

49. Ordonez, G.R., Puente, X.S., Quesada, V. \& LopezOtin, C. (2009). Proteolytic systems: Constructing degradomes. Methods in Molecular Biology 539, 33-47.

50. Anamika, K., Martin, J. \& Srinivasan, N. (2008). Comparative kinomics of human and chimpanzee reveal unique kinship and functional diversity generated by new domain combinations. BMC Genomics 9, 625 .

51. Tadepally, H.D., Burger, G. \& Aubry, M. (2008). Evolution of $\mathrm{C} 2 \mathrm{H} 2$-zinc finger genes and subfamilies in mammals: Species-specific duplication and loss of clusters, genes and effector domains. $B M C$ Evolutionary Biology 8, 176.

52. Moesta, A.K., Abi-Rached, L., Norman, P.J. \& Parham, P. (2009). Chimpanzees use more varied receptors and ligands than humans for inhibitory killer cell Ig-like receptor recognition of the MHCC1 and MHC-C2 epitopes. Journal of Immunology 182, 3628-3637.

53. Marvanova, M., Menager, J., Bezard, E., Bontrop, R.E., Pradier, L. \& Wong, G. (2003). Microarray analysis of nonhuman primates: Validation of experimental models in neurological disorders. FASEB Journal 17, 929-931.

54. Caceres, M., Lachuer, J., Zapala, M.A., Redmond, J.C., Kudo, L., Geschwind, D.H., Lockhart, D.J., Preuss, T.M. \& Barlow, C. (2003). Elevated gene expression levels distinguish human from nonhuman primate brains. Proceedings of the National Academy of Sciences of the USA 100, 13,03013,035 .

55. Lin, L., Liu, S., Brockway, H., Seok, J., Jiang, P., Wong, W.H. \& Xing, Y. (2009). Using high-density exon arrays to profile gene expression in closely related species. Nucleic Acids Research 37, e90.

56. Khaitovich, P., Lockstone, H.E., Wayland, M.T., Tsang, T.M., Jayatilaka, S.D., Guo, A.J., Zhou, J., Somel, M., Harris, L.W., Holmes, E., Pääbo, S. \& Bahn, S. (2008). Metabolic changes in schizophrenia and human brain evolution. Genome Biology $\mathbf{9}$, R124.

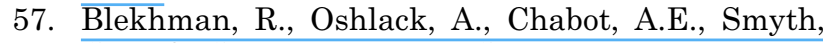
G.K. \& Gilad, Y. (2008). Gene regulation in primates evolves under tissue-specific selection pressures. PLoS Genetics 4, e1000271.

58. Uddin, M., Wildman, D.E., Liu, G., Xu, W., Johnson, R.M., Hof, P.R., Kapatos, G., Grossman, L.I. \& Goodman, M. (2004). Sister grouping of chimpanzees and humans as revealed by genome-wide phylogenetic analysis of brain gene expression profiles. Proceedings of the National Academy of Sciences of the USA 101, 2957-2962.

59. Arora, G., Polavarapu, N. \& McDonald, J.F. (2009). Did natural selection for increased cognitive ability in humans lead to an elevated risk of cancer? Medical Hypotheses 73, 453-456.

60. De Maria, A., Ugolotti, E., Rutjens, E., Mazza, S., Radic, L., Faravelli, A., Koopman, G., Di Marco, E.,
Costa, P., Ensoli, B., Cafaro, A., Mingari, M.C., Moretta, L., Heeney, J. \& Biassoni, R. (2009). NKp44 expression, phylogenesis and function in non-human primate NK cells. International Immunology 21, 245-255.

61. Soto, P.C., Stein, L.L., Hurtado-Ziola, N., Hedrick, S.M. \& Varki, A. (2010). Relative over-reactivity of human versus chimpanzee lymphocytes: Implications for the human diseases associated with immune activation. Journal of Immunology 184, 4185-4195.

62. Varki, A. (2008). Sialic acids in human health and disease. Trends in Molecular Medicine 14, 351-360.

63. Varki, N., Anderson, D., Herndon, J.G., Pham, T., Gregg, C.J., Cheriyan, M., Murphy, J., Strobert, E., Fritz, J. \& Else, J.G. (2009). Heart disease is common in humans and chimpanzees, but is caused by different pathological processes. Evolutionary Applications 2, 101-112.

64. Seidman, J.G. \& Seidman, C. (2002). Transcription factor haploinsufficiency: When half a loaf is not enough. Journal of Clinical Investigation 109, 451-455.

65. Gilad, Y., Oshlack, A., Smyth, G.K., Speed, T.P. \& White, K.P. (2006). Expression profiling in primates reveals a rapid evolution of human transcription factors. Nature, London 440, 242-245.

66. Nowick, K., Gernat, T., Almaas, E. \& Stubbs, L. (2009). Differences in human and chimpanzee gene expression patterns define an evolving network of transcription factors in brain. Proceedings of the National Academy of Sciences of the USA 106, 22,358-22,363.

67. Kasowski, M., Grubert, F., Heffelfinger, C., Hariharan, M., Asabere, A., Waszak, S.M., Habegger, L., Rozowsky, J., Shi, M., Urban, A.E., Hong, M.Y., Karczewski, K.J., Huber, W., Weissman, S.M., Gerstein, M.B., Korbel, J.O. \& Snyder, M. (2010). Variation in transcription factor binding among humans. Science 328, 232-235.

68. Torgerson, D.G., Boyko, A.R., Hernandez, R.D., Indap, A., Hu, X., White, T.J., Sninsky, J.J., Cargill, M., Adams, M.D., Bustamante, C.D. \& Clark, A.G. (2009). Evolutionary processes acting on candidate cis-regulatory regions in humans inferred from patterns of polymorphism and divergence. PLoS Genetics 5, e1000592.

69. Weis, E., Galetzka, D., Herlyn, H., Schneider, E. \& Haaf, T. (2008). Humans and chimpanzees differ in their cellular response to DNA damage and noncoding sequence elements of DNA repair-associated genes. Cytogenetic \& Genome Research 122, 92-102.

70. Farcas, R., Schneider, E., Frauenknecht, K., Kondova, I., Bontrop, R., Bohl, J., Navarro, B., Metzler, M., Zischler, H., Zechner, U., Daser, A. \& Haaf, T. (2009). Differences in DNA methylation patterns and expression of the CCRK gene in human and nonhuman primate cortices. Molecular Biology \& Evolution 26, 1379-1389.

71. Enard, W., Fassbender, A., Model, F., Adorján, P. Pääbo, S. \& Olek, A. (2004). Differences in DNA methylation patterns between humans and chimpanzees. Current Biology 14, R148-R149.

72. Bartel, D.P. (2004). MicroRNAs: Genomics, biogenesis, mechanism, and function. Cell 116, 281-297.

73. Cuellar, T.L. \& McManus, M.T. (2005). MicroRNAs and endocrine biology. Journal of Endocrinology 187, 327-332.

74. Miranda, K.C., Huynh, T., Tay, Y., Ang, Y.S., Tam, 
W.L., Thomson, A.M., Lim, B. \& Rigoutsos, I. (2006). A pattern-based method for the identification of microRNA binding sites and their corresponding heteroduplexes. Cell 126, 1203-1217.

75. Baev, V., Daskalova, E. \& Minkov, I. (2009) Computational identification of novel microRNA homologs in the chimpanzee genome. Computational Biology \& Chemistry 33, 62-70.

76. Brameier, M. (2010). Genome-wide comparative analysis of microRNAs in three non-human primates. BMC Research Notes 3, 64.

77. Cao, H., Yang, C.S. \& Rana, T.M. (2008). Evolutionary emergence of microRNAs in human embryonic stem cells. PLoS One 3, e2820.

78. Paz-Yaacov, N., Levanon, E.Y., Nevo, E., Kinar, Y., Harmelin, A., Jacob-Hirsch, J., Amariglio, N., Eisenberg, E. \& Rechavi, G. (2010). Adenosine-toinosine RNA editing shapes transcriptome diversity in primates. Proceedings of the National Academy of Sciences of the USA 107, 12,174-12,179.

79. Puente, X.S., Velasco, G., Gutierrez-Fernandez, A., Bertranpetit, J., King, M.C. \& Lopez-Otin, C. (2006). Comparative analysis of cancer genes in the human and chimpanzee genomes. BMC Genomics 7,15 .

80. Vamathevan, J.J., Hasan, S., Emes, R.D., AmrineMadsen, H., Rajagopalan, D., Topp, S.D., Kumar, V., Word, M., Simmons, M.D., Foord, S.M., Sanseau, P., Yang, Z. \& Holbrook, J.D. (2008). The role of positive selection in determining the molecular cause of species differences in disease. $B M C$ Evolutionary Biology 8, 273.

81. Watanabe, H., Fujiyama, A., Hattori, M., Taylor, T.D., Toyoda, A., Kuroki, Y., Noguchi, H., BenKahla, A., Lehrach, H., Sudbrak, R., Kube, M., Taenzer, S., Galgoczy, P., Platzer, M., Scharfe, M., Nordsiek, G., Blocker, H., Hellmann, I., Khaitovich, P., Pääbo, S., Reinhardt, R., Zheng, H.J., Zhang, X.L., Zhu, G.F., Wang, B.F., Fu, G., Ren, S.X., Zhao, G.P., Chen, Z., Lee, Y.S., Cheong, J.E., Choi, S.H., Wu, K.M., Liu, T.T., Hsiao, K.J., Tsai, S.F., Kim, C.G., OOta, S., Kitano, T., Kohara, Y., Saitou, N., Park, H.S., Wang, S.Y., Yaspo, M.L. \& Sakaki, Y. (2004). DNA sequence and comparative analysis of chimpanzee chromosome 22. Nature, London 429, 382-388.

82. Yu, G. (2009). An exon-based comparative variant analysis pipeline to study the scale and role of frameshift and nonsense mutation in the humanchimpanzee divergence. Comparative \& Functional Genomics 2009, 406-421.

83. Varki, A. (2001). Loss of $N$-glycolylneuraminic acid in humans: Mechanisms, consequences, and implications for hominid evolution. American Journal of Physical Anthropology 116, Suppl. 33, 54-69.

84. Hvilsom, C., Carlsen, F., Siegismund, H.R., Corbet, S., Nerrienet, E. \& Fomsgaard, A. (2008). Genetic subspecies diversity of the chimpanzee CD4 virusreceptor gene. Genomics 92, 322-328.

85. Stedman, H.H., Kozyak, B.W., Nelson, A., Thesier, D.M., Su, L.T., Low, D.W., Bridges, C.R., Shrager, J.B., Minugh-Purvis, N. \& Mitchell, M.A. (2004). Myosin gene mutation correlates with anatomical changes in the human lineage. Nature, London 428, 415-418.

86. Winter, H., Langbein, L., Krawczak, M., Cooper,
D.N., Jave-Suarez, L.F., Rogers, M.A., Praetzel, S. Heidt, P.J. \& Schweizer, J. (2001). Human type I hair keratin pseudogene $\varphi h H a A$ has functional orthologs in the chimpanzee and gorilla: Evidence for recent inactivation of the human gene after the Pan-Homo divergence. Human Genetics 108, 37-42.

87. Zhang, J., Webb, D.M. \& Podlaha, O. (2002). Accelerated protein evolution and origins of humanspecific features: FOXP2 as an example. Genetics 162, 1825-1835.

88. Konopka, G., Bomar, J.M., Winden, K., Coppola, G., Jonsson, Z.O., Gao, F., Peng, S., Preuss, T.M., Wohlschlegel, J.A. \& Geschwind, D.H. (2009). Human-specific transcriptional regulation of CNS development genes by FOXP2. Nature, London 462, 213-217.

89. Matlin, A.J., Clark, F. \& Smith, C.W. (2005). Understanding alternative splicing: Towards a cellular code. Nature Reviews Molecular Cell Biology 6, 386-398.

90. Grosso, A.R., Gomes, A.Q., Barbosa-Morais, N.L., Caldeira, S., Thorne, N.P., Grech, G., von Lindern, M. \& Carmo-Fonseca, M. (2008). Tissue-specific splicing factor gene expression signatures. Nucleic Acids Research 36, 4823-4832.

91. Glazko, G., Veeramachaneni, V., Nei, M. \& Makalowski, W. (2005). Eighty percent of proteins are different between humans and chimpanzees. Gene 346, 215-219.

92. Chimpanzee Sequencing and Analysis Consortium (2005). Initial sequence of the chimpanzee genome and comparison with the human genome. Nature, London 437, 69-87.

93. Lin, F.K., Pan, C.L., Yang, J.M., Chuang, T.J. \& Chen, F.C. (2009). CAPIH: A Web interface for comparative analyses and visualization of host-HIV protein-protein interactions. BMC Microbiology 9, 164.

94. King, M.C. \& Wilson, A.C. (1975). Evolution at two levels in humans and chimpanzees. Science 188, 107-116.

95. Varki, A. (2000). A chimpanzee genome project is a biomedical imperative. Genome Research 10, 10651070.

96. Cohen, J. (2007). Biomedical research. The endangered lab chimp. Science 315, 450-452.

97. Ollomo, B., Karch, S., Bureau, P., Elissa, N., Georges, A.J. \& Millet, P. (1997). Lack of malaria parasite transmission between apes and humans in Gabon. American Journal of Tropical Medicine \& Hygiene 56, 440-445.

98. Ferdowsian, H.R., Durham, D.L., Kimwele, C., Kranendonk, G., Otali, E., Akugizibwe, T., Mulcahy, J.B., Ajarova, L. \& Johnson, C.M. (2011). Signs of mood and anxiety disorders in chimpanzees. PLoS One 6 , e19855.

99. Anon. (2000). Chimpanzee Health Improvement, Maintenance, and Protection Act: Hearing before the Subcommittee on Health and Environment of the Committee on Commerce, House of Representatives, One Hundred Sixth Congress, second session, on H.R. 3514, 46pp. Washington, DC, USA: Committee on Commerce. Available at: http://www.gpo.gov/ fdsys/pkg/CHRG-106hhrg64771/pdf/CHRG-106 hhrg64771.pdf (Accessed 10.10.11). 